\title{
Polarization of Radiation from a Strongly Magnetized Accretion Disk: The Asymptotic Spectral Distribution
}

\author{
Yu. N. Gnedin ${ }^{1 *}$, N. A. Silant'ev ${ }^{1,2}$, and P. S. Shternin ${ }^{3}$ \\ ${ }^{1}$ Pulkovo Observatory, Russian Academy of Sciences, Pulkovskoe shosse 65, St. Petersburg, 196140 Russia \\ ${ }^{2}$ Instituto Nacional de Astrofísica, Óptica y Electrónica, Luis Enrique Erro No. 1, Apdo Postal 51 y 216 , \\ 72840 Tonantzintla, Puebla, México \\ ${ }^{3}$ St. Petersburg State Polytechnical University, ul. Politekhnicheskaya 29, St. Petersburg, 195251 Russia \\ Received June 7, 2005
}

\begin{abstract}
We calculate the polarization of the radiation from an optically thick accretion disk with a vertical averaged magnetic field. The polarization arises from the scattering of light by free electrons in a magnetized disk plasma. The Faraday rotation of the polarization plane during the propagation of a photon in a medium with a magnetic field is considered as the main effect. We discuss various models of optically thick accretion disks with a vertical averaged magnetic field. Our main goal is to derive simple asymptotic formulas for the polarization of radiation in the case where the Faraday rotation angle $\Psi \gg 1$ at the Thomson optical depth $\tau=1$. The results of our calculations allow the magnetic field strength in the region of the marginally stable orbit near a black hole to be estimated from polarimetric observations, including Xray observations expected in the future. Since the polarization spectrum of the radiation strongly depends on the accretion disk model, a realistic physical model of the accretion disk can be determined from data on the polarization of its radiation.
\end{abstract}

PACS numbers : 98.54.Aj; 98.54.Cm

DOI: $10.1134 / \mathrm{S} 1063773706010063$

Key words: compact objects, accretion disks, radiation polarization.

\section{INTRODUCTION}

An accretion disk is one of the main elements of the structure of accretion flows near compact objects-neutron stars, black holes, quasars, and active galactic nuclei (AGNs). Since there is no axial symmetry with respect to the line of sight, the total integrated radiation emerging from an accretion disk will be polarized during electron scattering. At present, the scattering by electrons is considered as the main mechanism of the polarization of optical radiation from accretion disks around compact objects and supermassive black holes. The Thomson scattering by electrons also makes a major contribution to the opacity in the optical range, producing a noticeable linear polarization. For AGNs and quasars, this conclusion is confirmed observationally, because the emission in broad lines of various elements is also polarized; in many cases, the polarization and the position angle are equal to those in the continuum (see Agol and Blaes 1996). The presence of a magnetic field leads to an additional effect-the Faraday rotation of the polarization plane along the photon

\footnotetext{
E-mail: gnedin@gao.spb.ru
}

mean free path in a scattering medium. As a result, a nontrivial spectral dependence of the polarization of radiation arises when the Faraday rotation angle at optical depth $\tau$ is large enough (Gnedin and Silant'ev 1997):

$$
\Psi=0.4\left(\frac{\lambda}{1 \mu \mathrm{m}}\right)^{2}\left(\frac{B}{1 \mathrm{G}}\right) \tau \cos \theta
$$

where $\lambda$ is the wavelength of the radiation and $\theta$ is the angle between the magnetic field direction and the line of sight.

Another possible source of polarization could be the synchrotron radiation of relativistic electrons in the disk itself or in a relativistic jet. However, for optical photons, this mechanism requires rather strong magnetic fields and high energies of relativistic electrons. Furthermore, the synchrotron radiation has a fairly high polarization (several tens of percent), with the degree of its polarization being wavelengthindependent. This is in conflict with the observations: once the dilution and interstellar polarization effects have been taken into account, the polarization of the optical radiation from compact objects and, in partic- 
ular, AGNs and quasars depends significantly on the wavelength.

This work is based on the solution of the Milne problem in a magnetized atmosphere. This problem was considered by a number of authors, who obtained numerical solutions (Silant'ev 1994, 2002; Agol and Blaes 1996; Agol et al. 1998; Shternin et al. 2003).

The main goal of this work is to derive simple asymptotic formulas for the polarization of radiation and to use them to analyze various models of accretion disks with a global magnetic field. In this case, it is more convenient to use the asymptotic formulas derived by Silant'ev (2002) for the Stokes parameters of the radiation emitted from a magnetized, optically thick plane-parallel atmosphere instead of numerical calculations.

\section{POLARIZATION OF THE RADIATION FROM AN OPTICALLY THICK ACCRETION DISK WITH A STRONG MAGNETIC FIELD}

Silant'ev (2002) derived simple analytical approximate formulas for the polarization of the radiation emerging from an optically thick accretion disk with a vertical averaged magnetic field,

$$
p_{l}(\mu)=\frac{1-g}{1+g} \frac{1-\mu^{2}}{J(\mu)} \frac{1}{\sqrt{(1-k \mu)^{2}+\delta^{2} \mu^{2}}},
$$

where $\mu=\cos \theta$ is the $\operatorname{cosine}$ of the angle between the magnetic field normal to the atmosphere (directed along the $z$ axis) and the direction of propagation of the radiation emerging from the disk toward the observer. The function $J(\mu)$ describes the angular distribution of the radiation emerging from the disk. This function as well as the numerical parameters $g$ and $k$ are given in Silant'ev (2002). The Faraday depolarization parameter can be introduced from Eq. (1),

$$
\delta=0.8\left(\frac{\lambda}{1 \mu \mathrm{m}}\right)^{2}\left(\frac{B}{1 \mathrm{G}}\right) .
$$

We consider the case where the electron scattering dominates in accretion disks $(k=0, g=0.83255)$.

Photons emerge mainly from a near-surface layer of the accretion disk with $\tau \approx 1$. If the Faraday rotation angle $\Psi$ that corresponds to this optical depth exceeds unity, the radiation emerging from the atmosphere will be depolarized through the addition of radiation fluxes with different rotation angles of the polarization plane. The Faraday rotation angle is too small for the radiation to be depolarized only for the directions perpendicular to the magnetic field. Of course, the diffusion of the radiation in the inner disk layers depolarizes it through multiple photon scattering even in the absence of a magnetic field.
However, the Faraday rotation enhances this effect. This implies that the polarization of the emergent radiation has a peaklike angular dependence with a peak in a direction perpendicular to the magnetic field. As the magnetic field strengthens, the peak width decreases. The angular size of the region in which the radiation is not completely depolarized is $\mu \sim 1 / \delta$.

Another important property of the polarized radiation from a magnetized atmosphere is a strong wavelength dependence of the polarization, which differs significantly from the case of classical Thomson scattering.

For strong magnetic fields (or for long wavelengths), when $\delta \mu \gg 1$, the simple asymptotic formula for the polarization takes the form

$$
p_{l}(B) \approx 0.09 \frac{1-\mu^{2}}{J(\mu) \mu} \frac{1}{\delta}
$$

Silant'ev (2002) also considered other model magnetic field distributions over the disk, e.g., purely radial and toroidal magnetic fields. In both cases, relations (2) and ( 4 ) take the same form with $\delta\left(1-\mu^{2}\right)^{1 / 2}$ substituted for $\delta \mu$. For a random magnetic field, the analytical asymptotic dependence does not change radically, resulting in $p_{l}(B) \sim 1 / \delta$.

In this paper, we restrict our analysis to an accretion disk with a nearly vertical averaged magnetic field. Other magnetic field distributions will be considered in separate papers.

\section{MODELS OF ACCRETION DISKS WITH A NEARLY VERTICAL AVERAGED MAGNETIC FIELD}

Let us briefly review the existing models of accretion disks with a vertical averaged magnetic field (see, e.g., Campbell 1997; Casse and Keppens 2002; Li 2002; Wang et al. 2003; Romanova et al. 2003; Pariev et al. 2003; Turner et al. 2003).

Campbell (1997) considered a dipole magnetic field. For radial distances from the gravitating center $r \gg z$, the magnetic field is represented as

$$
B_{z}=\frac{1}{2} B_{p}\left(\frac{R_{p}}{r}\right)^{3}, \quad B_{r} \sim \frac{z B_{z}}{r} .
$$

The spinup and spindown of magnetized stars with accretion disks and outflows were considered by Romanova et al. (2003). They performed calculations using the model of a three-dimensional magnetohydrodynamic disk accreting on an oblique dipole. The vertically averaged magnetic field was found to have a radial dependence $B_{z} \sim r^{-5 / 4}$. Using these results, the magnetic field can be estimated as

$$
B_{z} \approx 2.4 \times 10^{3}\left(\frac{10^{8} M_{\odot}}{M}\right)
$$




$$
\times\left(\frac{\dot{M}}{10^{26} \mathrm{~g} \mathrm{~s}^{-1}}\right)\left(\frac{r}{10 R_{\mathrm{g}}}\right)^{-5 / 4} \mathrm{G},
$$

where $M$ is the mass of the compact object (black hole), $\dot{M}$ is the accretion rate, and $R_{\mathrm{g}}$ is the gravitational radius.

$\mathrm{Li}$ (2002) obtained the following radial dependence of the vertically averaged magnetic field for a selfgravitating, magnetically supported disk:

$$
B_{z} \approx B_{r} \approx\left(\frac{2 \pi G \Sigma M}{R_{\mathrm{g}}^{2}}\right)^{1 / 2}\left(\frac{R_{\mathrm{g}}}{r}\right)
$$

where $\Sigma=\dot{M} / 2 \pi r v_{r}$ is the disk surface density. Assuming that the equipartition condition is satisfied, this leads to the radial dependence

$$
B_{z} \approx 10^{2}\left(\frac{10^{8} M_{\odot}}{M}\right)\left(\frac{\dot{M}}{10^{26} \mathrm{~g} \mathrm{~s}^{-1}}\right)^{1 / 2}\left(\frac{r}{10 R_{\mathrm{g}}}\right)^{-3 / 2} \text {. }
$$

Casse and Keppens (2002) constructed a model that depends on the parameters of the central disk region, more specifically, on the parameter $\beta=8 \pi P_{\text {gas }}(z=$ $0) / B^{2}(z=0)$. They derived the following formula for the vertically averaged magnetic field:

$$
B_{z} \approx B_{10} \frac{1}{\sqrt{\beta}}\left(\frac{10 R_{\mathrm{g}}}{r}\right)^{5 / 2} .
$$

Pariev et al. (2003) extended the classical ShakuraSunyaev approach to the model of a strongly magnetized accretion disk. They assumed that the radial dependence of the vertically averaged magnetic field obeys a power law,

$$
B_{z}=B_{10}\left(\frac{r}{10 R_{\mathrm{g}}}\right)^{-\eta},
$$

where $B_{10}$ is the magnetic field strength at $10 R_{\mathrm{g}}$ and $\eta>0$ is a constant. These authors performed numerical calculations and presented plots of the radial structure and radiation spectrum of the disk in the region where it is optically thick for four values of the basic parameters: $\eta=5 / 4, B_{10}=3 \times 10^{3} \mathrm{G} ; \eta=1$, $B_{10}=3 \times 10^{3} \mathrm{G} ; \eta=1.4, B_{10}=5 \times 10^{3} \mathrm{G} ; \eta=5 / 4$, $B_{10}=700 \mathrm{G}$, and for the mass of a supermassive black hole $M=10^{8} M_{\odot}$.

Liu et al. (2003) developed a model that was similar to the model by Pariev et al. (2003) and obtained the following expression for the average magnetic field:

$$
\begin{gathered}
B_{z}=7.2 \times 10^{4} \alpha_{01}^{-9 / 20} \beta_{1}^{-1 / 2} \\
\times\left(\frac{M}{10^{8} M_{\odot}}\right)^{-9 / 20}\left(\frac{\dot{M}}{0.1 \dot{M}_{E}}\right)^{2 / 5} r_{10}^{-51 / 40}
\end{gathered}
$$

Here, $\alpha$ is the viscosity in the Shakura-Sunyaev model, 0.1 , and $\dot{M}_{E}$ is the Eddington accretion rate, $\dot{M}_{E}=1.4 \times 10^{26} \times\left(M / 10^{8} M_{\odot}\right) \mathrm{g} \mathrm{s}^{-1}$. We see that the radial dependence of the average magnetic field is almost identical to the model by Pariev et al. (2003) with $\eta=5 / 4$.

Turner et al. (2003) considered an accretion disk model supported by radiation pressure. In their model, the vertically averaged magnetic field is represented as

$$
B_{z} \gtrsim 10^{8}\left(\frac{M_{\odot}}{M}\right)^{1 / 2}\left(\frac{r}{R_{\mathrm{g}}}\right)^{-\eta}
$$

where $\eta=3 / 4$.

The magnetic field was also included in the standard accretion disk models developed by Shakura and Sunyaev (1973) and Narayan and Yi (1995) (the ADAF model), but without a detailed definition of its geometry. For both models, $\eta=5 / 4$. For instance, Shakura and Sunyaev (1973) give the following formula for the magnetic field:

$$
\begin{gathered}
B_{z}=B_{10}\left(\frac{10 R_{\mathrm{g}}}{r}\right)^{51 / 40} \lesssim 6 \times 10^{7} \\
\times\left(\frac{M}{M_{\odot}}\right)^{-17 / 20}\left(\frac{\dot{M}}{10^{17} \mathrm{~g} \mathrm{~s}^{-1}}\right)^{2 / 5}\left(\frac{10 R_{\mathrm{g}}}{r}\right)^{51 / 40} .
\end{gathered}
$$

This implies that $B_{10} \equiv B_{10}(M, \dot{M})$ and $\eta=$ $51 / 40 \approx 5 / 4$.

The effective temperature of the disk can be determined from the relation

$$
\sigma T_{\mathrm{e}}^{4}(r)=\frac{3 G M \dot{M}}{8 \pi r^{3}}\left(1-\sqrt{\frac{3 R_{\mathrm{g}}}{r}}\right) .
$$

Let us calculate the Faraday depolarization parameter for the wavelength corresponding to the peak in the radiation spectrum of a given disk region. The disk radiates as a blackbody, implying that the wavelength corresponding to the peak in the radiation energy spectrum is defined as $\lambda=0.29 / T_{\mathrm{e}}$. The latter formula allows us to relate the radial distance from the compact object to the spectral wavelength corresponding to this distance:

$$
\frac{r}{10 R_{\mathrm{g}}}=1.3 \times 10^{2}\left(\frac{\lambda_{m}}{1 \mu \mathrm{m}}\right)^{4 / 3} M_{8}^{-1 / 3}\left(\frac{\dot{M}}{\dot{M}_{E}}\right)^{1 / 3} .
$$

As a result, we obtain the following for the Faraday rotation parameter:

$$
\delta=0.8\left(1.3 \times 10^{2}\right)^{-\eta}\left(\frac{\lambda_{m}}{1 \mu \mathrm{m}}\right)^{(6-4 \eta) / 3}
$$




$$
\times M_{8}^{\eta / 3}\left(\frac{\dot{M}}{\dot{M}_{E}}\right)^{-\eta / 3} B_{10} .
$$

Formula (16) can be rewritten as

$$
\begin{gathered}
\delta=0.8 \cdot(0.92)^{\eta} \times 10^{-13 \eta / 3} \\
\times\left(\frac{\lambda_{m}}{1 \mu \mathrm{m}}\right)^{(6-4 \eta) / 3}\left(\frac{M}{M_{\odot}}\right)^{2 \eta / 3} \\
\times\left(\frac{\dot{M}}{10^{17} \mathrm{~g} \mathrm{~s}^{-1}}\right)^{-\eta / 3} B_{10} .
\end{gathered}
$$

Strong depolarization arises only at the radiation wavelengths for which $\delta>1$.

\section{THE DEPOLARIZATION PARAMETER $\boldsymbol{\delta}$ FOR VARIOUS DISK MODELS}

Let us now calculate the Faraday depolarization parameter $\delta$ for various accretion disk models. We begin with the situation where the accretion disk is formed in close $\mathrm{X}$-ray binaries and consider two power laws of the magnetic field variation with radial distance: a dipole field with $\eta=3$ and a more popular case of $\eta=5 / 4$. For $\eta=3$, Eq. (17) yields

$$
\begin{gathered}
\delta=0.5 \times 10^{-11}\left(\frac{M}{10 M_{\odot}}\right)^{2} \\
\times\left(\frac{\dot{M}}{10^{17} \mathrm{~g} \mathrm{~s}^{-1}}\right)^{-1}\left(\frac{\lambda_{m}}{1 \mu \mathrm{m}}\right)^{-2} B_{10} .
\end{gathered}
$$

For optical ( $V$-band) photons,

$$
\delta \approx 2 \times 10^{-11}\left(\frac{M}{10 M_{\odot}}\right)^{2}\left(\frac{\dot{M}}{10^{17} \mathrm{~g} \mathrm{~s}^{-1}}\right)^{-1} B_{10},
$$

and an excessively strong magnetic field, $B>10^{11} \mathrm{G}$, is required for their depolarization. For $\mathrm{X}$-ray radiation with energy $E=1 \mathrm{keV}(\lambda=1.2 \AA)$, a magnetic field of $B \gtrsim 10^{4} \mathrm{G}$ produces a noticeable depolarization of the $\mathrm{X}$-ray photons emerging from an optically thick accretion disk.

For $\eta=5 / 4$ and the $V$ band of the radiation, the depolarization parameter is

$\delta=1.35 \times 10^{-5}\left(\frac{M}{10 M_{\odot}}\right)^{5 / 6}\left(\frac{\dot{M}}{10^{17} \mathrm{~g} \mathrm{~s}^{-1}}\right)^{-5 / 12} B_{10}$,

and the magnetic field strength at the inner disk radius, $B>10^{5} \mathrm{G}$, is large enough for a strong depolarization. The calculated magnetic fields that produce depolarization $(\delta>1)$ are given in Table 1 (the $V$ band and X-ray photons with an energy of $1 \mathrm{keV}$ ). The most surprising result arises for $\eta=3 / 2$. In this case, the depolarization parameter $\delta$ does not depend on the photon wavelength (energy), as in the classical Sobolev-Chandrasekhar case. Nevertheless, the degree of polarization is lower because of the depolarization effect.

We can predict that for $\eta>3 / 2$, harder photons will be depolarized more strongly than softer photons. In the opposite case, for $\eta<3 / 2$, soft photons will be depolarized more strongly and the polarization spectrum will have a cutoff at low energies.

\section{ASYMPTOTIC WAVELENGTH DEPENDENCE OF THE POLARIZATION \\ OF THE RADIATION FROM A MAGNETIZED ACCRETION DISK}

Finally, let us present our asymptotic formulas for the polarization using the analytical calculations by Silant'ev (2002).

Formula (4) cannot be used to determine the spectral dependence of the polarization of the radiation from a disk, because it is necessary to calculate the Stokes parameters of the radiation from the entire disk:

$$
\left\{\begin{array}{l}
F_{I} \\
F_{Q} \\
F_{U}
\end{array}\right\}=\int_{R_{\min }}^{R_{\max }} 2 \pi r d r \mu\left\{\begin{array}{c}
I \\
Q \\
U
\end{array}\right\},
$$

where $R_{\min }$ and $R_{\max }$ are the disk boundaries. The disk is assumed to be axially symmetric. The degree of polarization must then be calculated as

$$
p_{l}=\frac{\sqrt{F_{U}^{2}+F_{Q}^{2}}}{F_{I}} \text {. }
$$

The Stokes parameters $I, Q$, and $U$ are proportional to the radiation flux density at a given wavelength,

$$
F_{\lambda}=A_{\lambda} \frac{1}{\exp \left(\frac{2 \pi \hbar c}{k_{\mathrm{B}} T_{\mathrm{e}} \lambda}\right)-1} .
$$

If the temperature corresponding to the peak in the spectrum at the wavelength under consideration is observed far from the disk boundaries, i.e., if the radiation flux from the boundary regions of the disk is low at this wavelength, then the integration limits in (19) can be assumed to be $R_{\min } \rightarrow 0$ and $R_{\max } \rightarrow \infty$. In this case, the polarization spectrum of the observed radiation takes the following simple power-law form:

$$
p_{l}=\frac{0.09\left(1-\mu^{2}\right)}{J(\mu) \mu} C_{\eta}(15.3)^{\eta}\left(\frac{\lambda}{1 \mu \mathrm{m}}\right)^{(4 \eta-6) / 3}
$$


Table 1. Magnetic field strengths $B_{10}$ for various indices of the power-law radial dependence of the magnetic field in a standard accretion disk for $V$-band photons and X-ray photons $\left(E_{x} \approx 1 \mathrm{keV}\right)$ found from the condition $\delta>1$

\begin{tabular}{c|c|c|c|c|c|c|c|c}
\hline$\eta$ & 3 & $5 / 2$ & $3 / 2$ & 1.4 & $5 / 4$ & 1 & $3 / 4$ & 0 \\
\hline \multicolumn{7}{c}{$V$ band } \\
\hline$B_{10}>$ & $10^{11}$ & $1.2 \times 10^{9}$ & $5 \times 10^{6}$ & $2.5 \times 10^{5}$ & $10^{5}$ & $10^{4}$ & $5 \times 10^{3}$ & 5 \\
\hline \multicolumn{7}{c}{ X-ray photons } \\
\hline$B_{10}>$ & $10^{4}$ & $1.3 \times 10^{4}$ & $5 \times 10^{6}$ & $8 \times 10^{5}$ & $1.2 \times 10^{6}$ & $3 \times 10^{6}$ & $6 \times 10^{6}$ & $10^{8}$ \\
\hline
\end{tabular}

Table 2. Asymptotic wavelength dependence of the polarization for a standard magnetized accretion disk

\begin{tabular}{c|c|c|c|c|c|c|c|c}
\hline$\eta$ & 3 & $5 / 2$ & $3 / 2$ & 1.4 & $5 / 4$ & 1 & $3 / 4$ & 0 \\
\hline$p_{l}(\lambda) \sim$ & $\lambda^{2}$ & $\lambda^{4 / 3}$ & $\lambda^{0}$ & $\lambda^{-0.133}$ & $\lambda^{-1 / 3}$ & $\lambda^{-2 / 3}$ & $\lambda^{-1}$ & $\lambda^{-2}$ \\
\hline
\end{tabular}

$$
\times\left(\frac{\dot{M}}{\dot{M}_{E}}\right)^{\eta / 3} M_{8}^{-\eta / 3}\left(\frac{B_{10}}{1 \mathrm{G}}\right)^{-1} \sim \lambda^{(4 \eta-6) / 3},
$$

where $C_{\eta}$ is a constant that depends on the model parameter $\eta$ :

$$
C_{\eta}=\frac{\int_{0}^{\infty} \frac{x^{\eta+1} d x}{\exp \left(x^{3 / 4}\right)-1}}{\int_{0}^{\infty} \frac{x d x}{\exp \left(x^{3 / 4}\right)-1}} .
$$

The constant $C_{\eta}$ is plotted against the parameter $\eta$ in the figure. If the wavelength of interest is radiated predominantly from the regions close to the disk boundary, the parameter $C_{\eta}$ begins to depend on the radiation wavelength and the power-law polarization spectrum is distorted.

Thus, the asymptotic polarization spectrum allows us to determine the index of the power-law dependence of the average magnetic field for a standard magnetized disk. The results of our calculations for various accretion disk models are given in Table 2 .

Our main result presented in Table 2 is that the pattern of the spectral dependence of the polarization near the power-law index $\eta=1.5$ changes radically. For a stronger radial dependence of the magnetic field $(\eta>3 / 2)$, the polarization increases with wavelength. For a weaker radial dependence of the magnetic field $(\eta<3 / 2)$, the polarization increases in the short-wavelength range, i.e., $p_{l} \sim \lambda^{-n}$, where $n=$ $f(\eta)$. This implies that for a standard accretion disk, even the qualitative fact of the wavelength dependence of the polarization is indicative of the pattern of the radial dependence of the magnetic field in the disk.

\section{CONCLUSIONS}

We have presented formulas for the asymptotic spectral dependence of the polarization of the radiation from an optically thick accretion disk with a vertical averaged magnetic field. The polarization arises from the scattering of light by electrons in a magnetized disk plasma. We took into account the Faraday rotation of the polarization plane. We considered various models of optically thick accretion disks with a vertical average magnetic field. The spectral behavior of the polarization depends significantly on the type of power-law dependence of the magnetic field on the distance from the disk center. It turns out that for a stronger radial dependence of the magnetic field (a power-law index $\eta>1.5$ ), high-energy photons will be depolarized more strongly than radiation in a softer energy range. In contrast, for a weaker radial dependence of the magnetic field, soft photons will be depolarized more strongly, which will result in a cutoff in the polarization spectrum of the radiation in the long-wavelength range. This allows the powerlaw radial dependence of the magnetic field to be qualitatively estimated using only simple observations of the superscale wavelength dependence of the polarization.

\section{ACKNOWLEDGMENTS}

One us (P.S. Shternin) is grateful to the Dynasty Foundation and the International Center for Fundamental Physics (Moscow). This work was supported by the Russian Foundation for Basic Research (project no. 03-02-17223), the "Nonstationary Phenomena in Astronomy" program of the Presidium of the Russian Academy of Sciences, the "Extended Objects in the Universe" program of the Division of Physical Sciences of the Russian Academy of Sciences, and the "Program of the Ministry of Science and Education of Russia". 


\section{REFERENCES}

1. E. Agol and O. Blaes, Mon. Not. R. Astron. Soc. 282, 965 (1996).

2. E. Agol, O. Blaes, and C. Ionescu-Zanetti, Mon. Not. R. Astron. Soc. 293, 1 (1998).

3. F. Casse and R. Keppens, Astrophys. J. 581, 988 (2002).

4. C. G. Campbell, Magnetohydrodynamics in Binary Stars (Kluwer, Durdrecht, 1997).

5. Yu. N. Gnedin and N. A. Silant'ev, Basic Mechanisms of Light Polarization in Cosmic Media (Hartwood, Amsterdam, 1997).

6. Z.-Y. Li, Astrophys. J. 574, L159 (2002).

7. B. F. Liu, S. Mineshige, and K. Oshuga, Astrophys. J. 587, 561 (2003).

8. R. Narayan and I. Yi, Astrophys. J. 452, 710 (1995).

9. V. I. Pariev, E. G. Blackman, and S. A. Boldyrev, Astron. Astrophys. 407, 403 (2003).
10. M. M. Romanova, G. V. Ustyugova, A. V. Koldoba, et al., Astrophys J. 595, 1009 (2003).

11. N. I. Shakura and R. A. Sunyaev, Astron. Astrophys. 24, 3 (1973).

12. P. S. Shternin, Yu. N. Gnedin, and N. A. Silant'ev, Astrofizika 46, 433 (2003) [Astrophys. 46, 350 (2003)].

13. N. A. Silant'ev, J. Quant. Spectrosc. Radiat. Transf. 52, 207 (1994).

14. N. A. Silant'ev, Astron. Astrophys. 383, 326 (2002).

15. N. J. Turner, J. M. Stone, J. H. Krolik, and T. Sano, astro-ph/0304511 (2003).

16. D.-X. Wang, R.-Yi. Ma, and W.-H. Lei, Astrophys. J. 595, 109 (2003).

Translated by G. Rudnitskiǔ 